\title{
A Different Approach to Assess Oxidative Stress in Dengue Hemorrhagic Fever Patients Through
} the Calculation of Oxidative Stress Index

\author{
Edi Hartoyo ${ }^{1,2}$, Iskandar Thalib ${ }^{1,3}$, Cynthia Maharani Puspita Sari ${ }^{3}$, Windy Yuliana Budianto ${ }^{4}$, Eko Suhartono ${ }^{5}$ \\ ${ }^{1}$ Department of Child Health, Faculty of Medicine, Lambung Mangkurat University, Banjar Baru, Indonesia \\ ${ }^{2}$ Department of Child Health, Ulin Public Hospital, Banjarmasin, Indonesia \\ ${ }^{3}$ Research and Development Unit, Mutiara Bunda Mother and Child Hospital, Martapura, Indonesia \\ ${ }^{4}$ Department of Pediatric Nursery, Faculty of Medicine, Lambung Mangkurat University, Banjar Baru, Indonesia \\ ${ }^{5}$ Department of Medical Chemistry/Biochemistry, Faculty of Medicine, Lambung Mangkurat University, Banjar Baru, Indonesia
}

\section{ABSTRACT}

The objectives of this study were to determine the involvement of Oxidative Stress (OS) in the pathogenesis of dengue hemorrhagic fever (DHF) through the analysis of oxidative stress Index (OSI). The levels of malondialdehyde (MDA), superoxide dismutase (SOD) and catalase (CAT) activity, and OSI were measured in 61 child dengue patients and (aged 6 months-18 years) with three different stages of DHF, i.e stage I, II, and III. The results show that the levels of MDA, SOD and CAT activity, and OSI significantly different between the group. The all parameters that investigated in this present study seems higher MDA level and OSI in the higher grade of DHF, except for SOD and CAT activity. From this result, it can be concluded that oxidative stress pathways might be involved in the pathomechanism of DHF and OSI might be used as a biomarker for OS and the severity in DHF patients.

Keywords: Dengue hemorrhagic fever, oxidative stress, oxidative stress index

\section{INTRODUCTION}

Dengue hemorrhagic fever (DHF) is a major public health problem worldwide with a rapidly increased incidence and spread during the past five decades [1]. Concerning the incidence of DHF all over the world, the graph has risen up noticeably in recent decades and over $40 \%$ of the world's population is now at risk from DHF. It has been estimated that there may be 50 to 100 million dengue infections globally per year [2].

DHF has been reported to be the leading cause of hospitalizations and death among children in several countries in Southeast Asia, including Indonesia [1]. The first recorded outbreak of a dengue disease compatible with DHF occurred in Surabaya in 1968 with a mortality rate of $41,3 \%$. Since then, major outbreaks of DHF with significant mortality have occurred in most of the regions in Indonesia [3].

${ }^{*}$ Corresponding author:

Eko Suhartono

Department of Medical Chemistry/Biochemistry, Faculty of Medicine, Lambung Mangkurat University

Jalan Ahmad Yani Km. 36, Banjarbaru, Indonesia 70712

Email: ekoantioxidant@gmail.com
Dengue is a mosquito-borne viral disease caused by one of the four serotypes of dengue viruses, which are transmitted to humans through the bites of infected female Aedes mosquitoes, with Aedes aegypti being the principal vector [4]. Four distinct serotypes of dengue virus are known as DEN-1, DEN-2, DEN-3 and DEN-4 [2]. WHO classified this disease into several class according to the symptoms and severity. Symptomatic dengue virus infections were grouped into three categories: undifferentiated fever, dengue fever $(\mathrm{DF})$ and dengue hemorrhagic fever (DHF). DHF had more classified into four severity grades, with grades III and IV being defined as dengue shock syndrome (DSS) [5].

The pathogenesis of DHF is poorly understood. Secondary infections with multiple serotypes were found as one of the risk factors for developing DHF by a phenomenon called antibody-dependent enhancement,

\section{How to cite:}

Hartoyo E, Thalib I, Sari CMP et al. (2017) A Different Ap-

proach to Assess Oxidative Stress in Dengue Hemorrhagic Fever Patients Through the Calculation of Oxidative Stress

Index. J. Trop. Life. Science 7 (3): $237-242$. 
which leads to high viremic state. However, the mechanism behind this high viremic status causing pathology of DHF is still not clear [6]. In this respect, it has been proposed the involvement of DENV infection-derived oxidative stress (OS) on the severity of dengue. This is based on their ability to trigger the release of pro-inflammatory cytokines, including TNF-alpha, participating in collective action in the immunopathogenesis of dengue disease [7]. By definition, OS is a disturbance in the balance between the production of reactive oxygen species (ROS) and antioxidants defenses in favor of the pro-oxidants [8]. Our previous study clearly indicated that OS might be involved in pathomechanism of DHF and it seems is higher with the higher grade of DHF [9].

Recently, OS not only evaluated by analyze the level of oxidants and antioxidants, but also analyze through a proportion or ratio [10]. This ratio was first proposed by Sharma et al. and known as OS index (OSI) [11]. OSI is a ratio between Total Oxidants Status (TOS) and Total Antioxidant Status (TAS) [12-13]. Generally, TOS and TAS was measured by the method of Erel. This present study used a different approach. OSI is calculated by measuring the ratio between MDA level and the total activity of superoxide dismutase (SOD) and catalase (CAT).

Considering the OS mechanism involved in DHF, this present study is aimed to assess the level of OS in several classes of DHF through measurement OSI. The results are expected to be used as supplementary information how the OS pathomechanism is involved in several pathological conditions in DHF. Also, the results of this study are expected to serve as information to define a new marker for several pathological conditions in DHF.

\section{MATERIALS AND METHODS Subjects}

The study was conducted on DHF patients after informed consent was obtained from them. It was approved by the Ethics Commission of the Faculty of Medicine, Lambung Mangkurat University, Banjarmasin, South Kalimantan, Indonesia. A total of 61 subjects (aged 6 months -18 years) were recruited for this study. Subjects were divided into 3 groups: group I (DHF-1, n $=22$ ) with a grade I of DHF, while group II (DHF-2, $n$ = 36) with grade II of DHF, and group III (DHF-3, n = 3) with grade III of DHF according to the World Health Organization (WHO) 1997 guidelines for control and prevention of DHF.11 Dengue infection was confirmed by IgM/IG g and NS-1 detection.

\section{Samples collection}

Samples of blood were collected from the patients using vacutainers containing EDTA. The samples were centrifuged for 15 minutes at $2000 \mathrm{rpm}$ and stored at $20^{\circ} \mathrm{C}$ until further analysis. All parameters analysis were conducted at Medical Chemical/Biochemical Laboratory of Lambung Mangkurat University, Banjarbaru, South Kalimantan, Indonesia.

\section{MDA level analysis}

MDA levels was calculated by thiobarbituric acid reactive substances (TBARS) by the technique already proposed by Buege and Aust [14]. The supernatant was put into a pyrex tube that contained $10 \%$ of trichloracetic acid and $0.67 \%$ of thiobarbituric acid and incubated at $100^{\circ} \mathrm{C}$ for 15 minutes. Then chill the mixture on ice for 5 minutes and add the $1.5 \mathrm{~mL}$ of $\mathrm{n}$-butyl-liquor. Let the mixture stand for 40 seconds and centrifuged at 1000 rpm for 15 minutes. The TBARS value was calculated by the spectrophotometer at the absorbance of $532 \mathrm{~nm}$ and figured utilizing the coefficient $1.56 \times 105 \mathrm{~mol} / \mathrm{cm}$. The MDA concentration expressed in $\mu$ mol MDA. As a standard solution, we used commercially MDA.

\section{SOD activity analysis}

The activity of superoxide dismutase (SOD) was measured according to Misra and Fridovich [2]. The oxidation of epinephrine was followed in terms of the production of adrenochrome, which exhibits an absorption maximum at $480 \mathrm{~nm}$. The reaction mixture contains 0,1 $\mathrm{M}$ epinephrine, $0.1 \mathrm{mM}$ EDTA and carbonate buffer ( $\mathrm{pH}$ 10.2). The temperature was maintained at $30^{\circ} \mathrm{C}$ and the absorbance measured at $480 \mathrm{~nm}$ using a spectrophotometer. One unit of SOD activity was defined as the enzyme amount that inhibits the rate of epinephrine oxidation by $50 \%$ [15].

\section{CAT activity analysis}

CAT activity estimated by using Aebi's method. The first step was to prepare the stock solution by using 2 $\mathrm{mL}$ and $1 \mathrm{~mL}$ from phosphate buffer at $\mathrm{pH} 7$ and $\mathrm{H}_{2} \mathrm{O}_{2}$ (30 mM) respectively. Then, $50 \mu \mathrm{L}$ of the lysate was added to the stock solution. The ability of catalase to work a reducing factor was measured by determining the changes in absorbance at $240 \mathrm{~nm}$ [16].

\section{OSI analysis}

The OSI was a ratio between MDA and the total activity of SOD and CAT. It was calculated following to equation: 


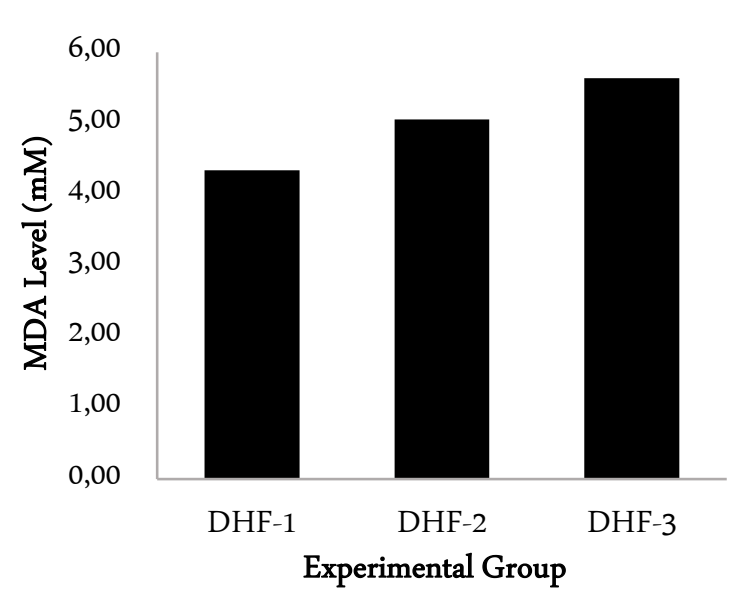

Figure 1. Comparison of MDA level in the different grade of DHF. DHF-1: grade I DHF; DHF-2: grade II DHF; and DHF-3: grade III DHF

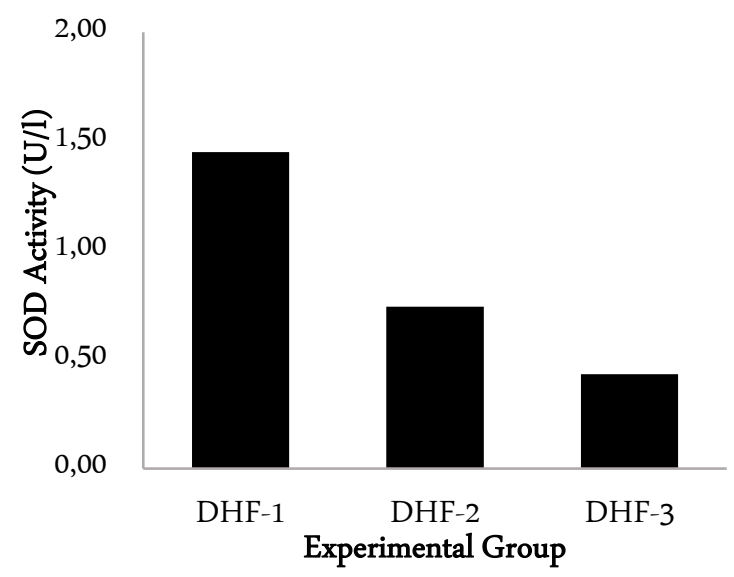

Figure 2. Comparison of SOD activity in the different grade of DHF. DHF-1: grade I DHF; DHF-2: grade II DHF; and DHF-3: grade III DHF

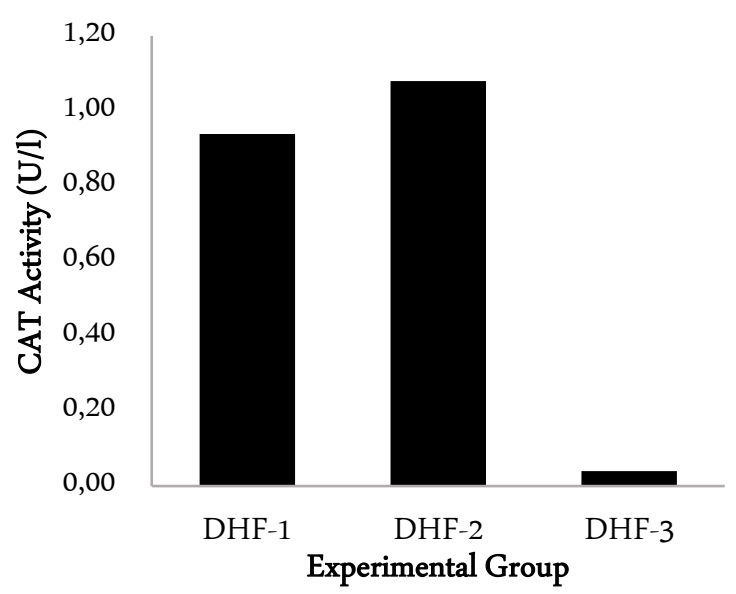

Figure 3. Comparison of CAT activity in the different grade of DHF. DHF-1: grade I DHF; DHF-2: grade II DHF; and DHF3: grade III DHF

$$
O S I(\mu M / \text { unit })=\frac{M D A}{S O D+C A T}
$$

\section{Statistical analysis}

The results were expressed as mean \pm SE for three replicates. Significance of mean differences of all MDA, SOD, CAT, and OSI between groups were statistically compared using one-way Analysis of Variance (ANOVA) or Kruskal-Wallis test and followed by a post hoc Tukey's Honestly Significant Difference (HSD) or Mann-Whitney test for multiple range test. Significance was set at $\mathrm{p}<0.05$. The software used for the data analysis were the Statistical Package for the Social Sciences (SPSS) version 16.0 and Microsoft Excel 2010 for Windows Vista.

\section{RESULTS AND DISCUSSION}

In this present study, OS status in the different grade of DHF patients was evaluated by measuring OSI. To obtain the level of OSI, MDA level, SOD, and CAT activity must calculate. The results show in Figure 1 3. According to Figure 1, MDA level seems to increase with the increasing of DHF grade, and from Figure 2 and 3, both SOD and CAT activity seems to decrease with the increasing of DHF grade. Kruskal-Wallis test results show that the MDA level is significantly different from each other $(p<0.05)$. Mann-Whitney test results show that there are significant differences in MDA level between all group of treatments $(p<0.05)$ (Table 1$)$. For the SOD and activity, Kruskal-Wallis test results show that the both SOD and CAT activity are significantly different from each other $(p<0.05)$. Mann-Whitney test results show that there are significant differences in SOD and CAT activity level among all group of treatments, except between DHF-1 and DHF-2 in CAT activity $(\mathrm{p}<0.05)$ (Table 1$)$.

After determining the MDA level, and SOD and CAT activity, the OSI can be obtained. The results show in Figure 4. OSI seems to increase with the increasing of DHF grade (Figure 4). Kruskal-Wallis test results shows that the OSI level is significantly different from each other $(\mathrm{p}<0.05)$. Mann-Whitney test results show that there are significant differences between all group of treatments (Table 1).

Recently, it has been indicated that many of infections, including viral infection like dengue virus trigger the production of ROS $[6,17]$. In dengue infection, proinflammatory cytokines secreted during the early phase could contribute to oxidative stress, which may contribute to the pathogenesis. Again, in this present study, we observed an alteration in redox status 
Table 1. Mann-Whitney test results of MDA level, SOD and CAT activity, and OSI in the different grade of DHF patients

\begin{tabular}{ccccc}
\hline \multirow{2}{*}{ Group comparison } & \multicolumn{5}{c}{ p-value } \\
\cline { 2 - 5 } & $M D A$ & $S O D$ & $C A T$ & $O S I$ \\
\hline DHF-1 : DHF-2 & 0.000 & 0.000 & 0.564 & 0.000 \\
\hline DHF-1 : DHF-3 & 0.006 & 0.009 & 0.006 & 0.006 \\
\hline DHF-2 : DHF-3 & 0.007 & 0.032 & 0.004 & 0.004 \\
\hline
\end{tabular}

Note: *)DHF-1: grade I DHF; DHF-2: grade II DHF; and DHF-3: grade III DHF. Results presented as mean \pm SD. $\mathrm{p}$-Values were calculated using the Kruskal Wallis test and followed by MannWhitney test; $\mathrm{p}<0.05$ was considered statistically significant.

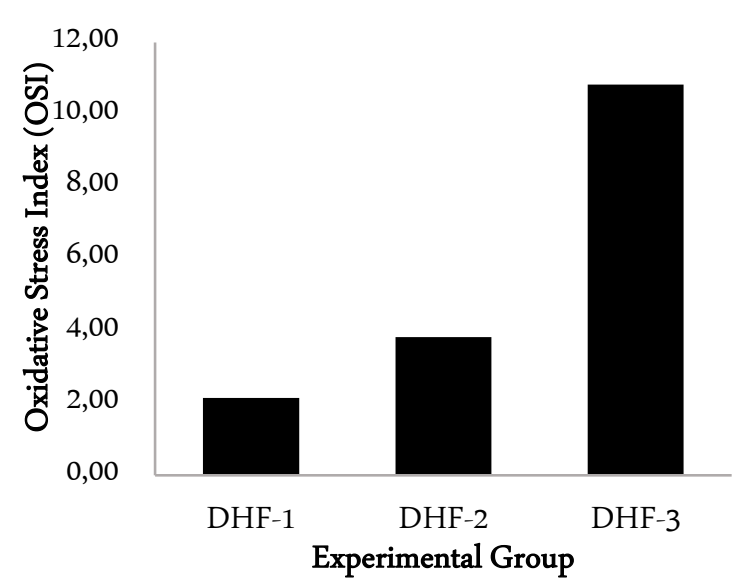

Figure 4. Comparison of OSI in the different grade of DHF. DHF-1: grade I DHF; DHF-2: grade II DHF; and DHF-3: grade III DHF

in the different clinical spectrum of dengue viral infection by calculated the OSI but with a different approach.

OSI in this study was calculated as the ratio between MDA and the total of SOD and CAT activity. To calculated the OSI, firstly we evaluated the MDA level. The results show that in the grade III of DHF, the level of MDA was found significantly different with both grade I and II. Similar changes were found in the levels of MDA among different grade of DHF. Among DHF cases, MDA levels were significantly different from each other, which is similar to our previous report [9]. Other parameters that we evaluated before the calculation of OSI were SOD and CAT activity. Both SOD and CAT activity seems to be decreased with the increasing of DHF grades. To best of our knowledge, this is the first report stating the alteration in redox status among the different grades of DHF.

After the calculation of MDA level, and SOD and CAT activity, the OSI could calculate. The result shows that the OSI were significantly different from each other. The OSI were increased with the increasing of DHF severity. It is consistent with several previous reports that the increasing level of oxidative stress might be related to the severity of dengue viral infection and may be involved in the pathogenesis of its complications $[6,7]$.

All the results of this present study suggested the involvement of OS in DHF which is an agreement with some previous results. The basic mechanism can be explained in two different ways, i.e.:

1) Dengue virus could induce a process known as respiratory burst. Respiratory burst is a term used when neutrophils become activated through the actions of NADPH oxidase and generate ROS production that can be very effective to kill a microorganism, inducing virus [9].

2) This OS is a result of the interaction between adhesion molecules on both phagocyte and vascular endothelial cell surfaces in the sites of tissue inflammation during dengue infection [18].

3) OS in DHF patients could be formed via the formation of heme oxygenasi-1 (HO-1) by the monocytes. It is based on a recent investigation compared HO-1 production by monocytes in vivo in various acute inflammatory illnesses and in healthy controls. Significantly elevated HO-1 mRNA levels seen in acute inflammatory illnesses suggest that monocyte production of HO-1 serves as a potent anti-inflammatory agent in controlling excessive cell or tissue injury in the presence of oxidative stress and cytokinemia [19].

4) Dengue virus infection could interfere the intracellular iron metabolism; this may trigger the Fenton reaction, thus producing hydroxyl radicals, which are highly toxic to cells. It is based on a previous result which observed the ROS formation and cell viability during dengue infection treat with a membrane permeable iron chelator. The result shows that treatment with this iron chelator could reduce ROS formation and increase cell viability during the dengue infection [20].

5) The dengue infection could affect the antioxidant enzymatic-like SOD, CAT, and GSH. However, the exact mechanism on the matter remains unclear, but the previous study reported that SOD, CAT, and antioxidative serum capacity was found lower in dengue fever patients than control [21].

However further studies are needed to determine 
which one is an exact mechanism for the increasing of ROS and OS in DHF patients. Furthermore, MDA level was found significantly increased in all stage of DHF that we investigated in this present study. The increasing of MDA level is caused by an OS

condition. The ROS can attack polyunsaturated fatty acids and initiate lipid peroxidation, a process that can eventually lead to a loss of membrane function and its integrity in DHF [6]. One of the final products of lipid peroxidation is MDA which is measured in this study

\section{CONCLUSION}

In conclusion, the present study demonstrated that OS may play a role in the pathogenesis of DHF which can be seen from the level of MDA, the activity of SOD and CAT, and OSI. Also, the present study demonstrated can be used as reasonable biomarkers for early prediction of severity of viral infection particularly in a case of DHF.

\section{ACKNOWLEDGMENT}

\section{REFERENCES}

1. Sitepu MS, Kaewkungwal J, Luplerdlop N et al. (2013) Temporal patterns and a disease forecasting model of dengue hemorrhagic fever in Jakarta based on 10 years of surveillance data. The Southeast Asian Journal of Tropical Medicine and Public Health 44 (2): 206-217.

2. Kumar CM, Vyas KSK, Krishna YS (2017) Clinical profile of dengue fever with severe thrombocytopenia and its complications: A retrospective study at a tertiary care hospital in South India. International Journal of Research in Medical Sciences 5 (5): $1-5$. doi: $10.18203 / 2320-$ 6012.ijrms20171543.

3. Yee LY, Heryaman H, Faridah L (2017) The relationship between frequency of fogging focus and incidence of dengue hemorrhagic fever cases in Bandung in year 2010 - 2015. International Journal of Research in Medical Sciences 4 (2): 456 - 459. doi: 0.18203/2394-6040.ijcmph20170272.

4. Kamaruddin D, Sungkar S (2013) The trend of dengue hemorrhagic fever cases in Central Jakarta 2008-2010. The trend of dengue hemorrhagic fever. eJournal Kedokteran Indonesia. 1 (1): 15 - 22. doi: 10.23886/ejki.1.1606.15-22.

5. Naseer F, Nazish A, Khan R et al. (2015) Dengue: An escalating problem of our society. Open Journal of Epidemiology 5 (1): 9 - 13. doi: 10.4236/ojepi.2015.51002.

6. Soundravally R, Sankar P, Bobby Z, Hoti SL (2008) Oxida- tive stress in severe dengue viral infection: Association of thrombocytopenia with lipid peroxidation. Platelets 19 (6): 447 - 454. 10.1080/09537100802155284.

7. Castro R, Pinzon HS, Alvis-Guzman N (2015) A systematic review of observational studies on oxidative/nitrosative stress involvement in dengue pathogenesis. Colombia Medica 46 (3): $135-143$.

8. Castro-Orozco R, Alvis-Guzman NR (2016) Role of oxidative and nitrosative stress in dengue pathogenesis: A minireview. Journal of Molecular and Genetic Medicine 10: 229. doi: 10.4172/1747-0862.1000229.

9. Hartoyo E, Thalib I, Suhartono E, Yunanto A (2016) Oxidative and chlorinative stress in children with dengue hemorrhagic fever. International Journal of Pharmaceutical and Clinical Reserach 8 (8): 1186 - 1191.

10. Kania N, Thalib I, Suhartono E. (2016) Chlorinative index in liver toxicity induced by iron. International Journal of Pharmaceutical and Clinical Reserach 8 (9): 1300 - 1304.

11. Udipi S, Ghugre P, Gokhale C (2012) Iron, oxidative stress and health. In: Dr. Volodymyr Lushchak eds. Oxidative stress - Molecular mechanisms and biological effects. doi: 10.5772/34975.

12. Allameh A, Amini-Harandi A, Osati-Ashtiani F, O’Brien PJ (2008) Iron overload induced apoptotic cell death in isolated rat hepatocytes mediated by reactive oxygen species. Iranian Journal of Pharmaceutical Research 7 (2): 115 - 121.

13. Sevcikova M, Modra H, Slaninova A, Svobodova Z (2011) Metals as a cause of oxidative stress in fish: A review. Veterinarni Medicina 56 (11): 537 - 546.

14. Suhartono E, Triawanti, Leksono AS, Djati MS (2015) Effects of cadmium exposure on lipid peroxidation and chlorinative stress of rat kidney. The Journal Experimental Life Science 5 (1): 1 - 5. doi: 10.21776/ub.jels.2015.005.01.01.

15. Suhartono E, Iskandar, Hamidah S, Arifin YF (2015) Phytochemical constituents analysis and neuroprotective effect of leaves of Gemor (Nothaphoebe coriacea) on cadmium-induced neurotoxicity in rats: An in-vitro study. International Journal of Toxicological and Pharmacological Research 7 (6): $297-302$.

16. Lestarisa T, Alexandra FD, Jelita H, Suhartono E (2106) Peroxidative index: A new marker in kidney toxicity induced by mercury. Journal of Toxicological and Pharmacological Research 8: $440-444$.

17. Ivanov AV, Bartosch B, Isaguliants MG (2107) Oxidative stress in infection and consequent disease. Oxidative Medicine and Cellular Longevity 2017 (2017). doi: $10.1155 / 2017 / 3496043$. 
18. Seet RCS, Lee CYJ, Lim ECH et al. (2009) Oxidative damage in dengue fever. Free Radical Biology and Medicine 47 (4): 375 - 380. doi: 10.1016/j.freeradbiomed.2009.04.035.

19. Gill L, Martinez G, Tapanes R, Castro O, Gonzalez D, Bernardo L, Vasquez S, Kouri G, Guzman M (2004) Oxidative stress in adult dengue patients. The American Society of Tropical Medicine and Hygiene 71 (5): 652 - 657.

20. Suwanprinya L, Morales NP, Sanvarinda P et al. (2016) Den- gue virus-induced reactive oxygen species production in rat microglia cells. Japanese Journal of Infectious Diseases 70 (4): 383 - 387. doi: 10.7883/yoken.JJID.2016.236.

21. Rasool M, Malik A, Khan KM et al. (2015) Assessment of biochemical and antioxidative status in patients suffering from dengue fever. Journal of Huazhong University of Science and Technology 35 (3): 411 - 418. doi: 10.1007/s11596015-1446-x. 